\title{
Stable witness-beam formation in a beam-driven plasma cathode
}

A. Knetsch $\odot,{ }^{1,}{ }^{*}$ B. Sheeran, ${ }^{1,2}$ L. Boulton, ${ }^{1,3,4}$ P. Niknejadi, ${ }^{1}$ K. Põder, ${ }^{1}$ L. Schaper, ${ }^{1}$ M. Zeng, ${ }^{1}$ S. Bohlen,${ }^{1,2}$ G. Boyle, ${ }^{1}$ T. Brümmer ${ }^{1}$ J. Chappell,${ }^{5}$ R. D'Arcy, ${ }^{1}$ S. Diederichs,${ }^{1,2}$ B. Foster, ${ }^{6}$ M. J. Garland, ${ }^{1}$ P. Gonzalez Caminal, ${ }^{1,2}$ B. Hidding, ${ }^{3,4}$ V. Libov, ${ }^{1}$ C. A. Lindstrøm, ${ }^{1}$ A. Martinez de la Ossa, ${ }^{1}$ M. Meisel,,${ }^{1,2}$ T. Parikh, ${ }^{1}$ B. Schmidt, ${ }^{1}$ S. Schröder, ${ }^{1,2}$ G. Tauscher, ${ }^{1,2}$

\author{
S. Wesch, ${ }^{1}$ P. Winkler, ${ }^{1,2}$ J. C. Wood, ${ }^{1}$ and J. Osterhoff ${ }^{1}$ \\ ${ }^{1}$ Deutsches Elektronen-Synchrotron DESY, Notkestr. 85, 22607 Hamburg, Germany \\ ${ }^{2}$ University of Hamburg, Hamburg, Germany \\ ${ }^{3}$ SUPA, Department of Physics, University of Strathclyde, Glasgow, United Kingdom \\ ${ }^{4}$ The Cockcroft Institute, Daresbury, United Kingdom \\ ${ }^{5}$ University College London, London, United Kingdom \\ ${ }^{6}$ John Adams Institute for Accelerator Science at University of Oxford, Oxford, United Kingdom
}

(Received 1 July 2021; accepted 20 September 2021; published 7 October 2021)

\begin{abstract}
Electron beams to be accelerated in beam-driven plasma wakes are commonly formed by a photocathode and externally injected into the wakefield of a preceding bunch. Alternatively, using the plasma itself as a cathode offers the possibility of generating ultrashort, low-emittance beams by trapping and accelerating electrons from the ambient plasma background. Here, we present a beam-driven plasma cathode realized via laser-triggered density-downramp injection, showing stable beam formation over more than a thousand consecutive events with an injection probability of $95 \%$. The plasma cathode is highly tunable, resulting in the injection of electron bunches of tens of $\mathrm{pC}$ of charge, energies of up to $79 \mathrm{MeV}$, and relative energy spreads as low as a few percent. The stability of the injected beams was sufficiently high to experimentally determine their normalized emittance of $9.3 \mu \mathrm{m}$ rms with a multishot method.
\end{abstract}

DOI: $10.1103 /$ PhysRevAccelBeams.24.101302

\section{INTRODUCTION}

Plasma wakes excited by charged particle beams [1-3] are capable of generating $\mathrm{GV} / \mathrm{m}$ accelerating gradients $[4,5]$ — orders of magnitude higher than those produced by conventional radio-frequency-based technologies, thereby promising a drastic reduction in both the size and cost of particle-accelerator facilities. Such a decrease is of particular significance for future linear colliders [6] and the provision of compact free-electron-laser photon sources. The research field of beam-driven plasma-wakefield acceleration (PWFA) is dynamic, with advances ranging from the energy doubling of a $42 \mathrm{GeV}$ electron beam over less than a meter [7] to the efficient energy transfer from the beam driving the wake (the drive beam) to the trailing beam experiencing the accelerating field (the witness beam) [8-11]. Witness beams can be injected into the plasma wake originating either from an external source, e.g., a photocathode, or internally by the trapping of ambient

*alexander.knetsch@desy.de

Published by the American Physical Society under the terms of the Creative Commons Attribution 4.0 International license. Further distribution of this work must maintain attribution to the author(s) and the published article's title, journal citation, and DOI. plasma electrons. Research on external injection methods has predominantly concentrated on maximizing energy transfer from the drive to the witness beam while preserving other beam parameters, such as energy spread [11,12]. Internal injection methods have the potential to provide witness beams with charges of tens to hundreds of picocoulombs, femtosecond bunch durations, and normalized transverse-emittance values much smaller than their drive beams [13-18]. Once injected, the phase-stable acceleration inherent to PWFAs allows for large single-stage energy gains [19] while the near-luminal phase velocity of the wake ensures a relatively high injection threshold and therefore maintains a low dark-current level, which could otherwise increase the emittance [20]. Beam-driven internal injection therefore offers the opportunity to generate a new class of beams with significantly enhanced brightness in comparison to conventional accelerator sources - an extremely desirable feature for future photon sources and applications in highenergy physics. While a variety of complementary internalinjection methods have been shown to work in principle [21-23], precise control over the injection process, and consequently the injected witness-beam parameters, has so far remained relatively unexplored.

Density-downramp injection (DDI) [24-29] is considered to be one of the most promising methods to generate high-brightness beams from PWFAs [30]—a prediction 


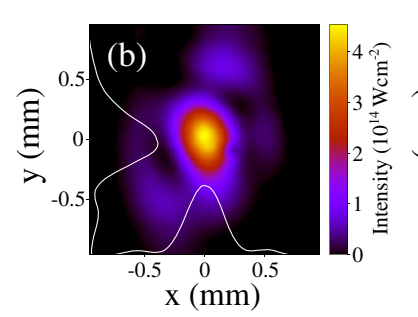

(a)
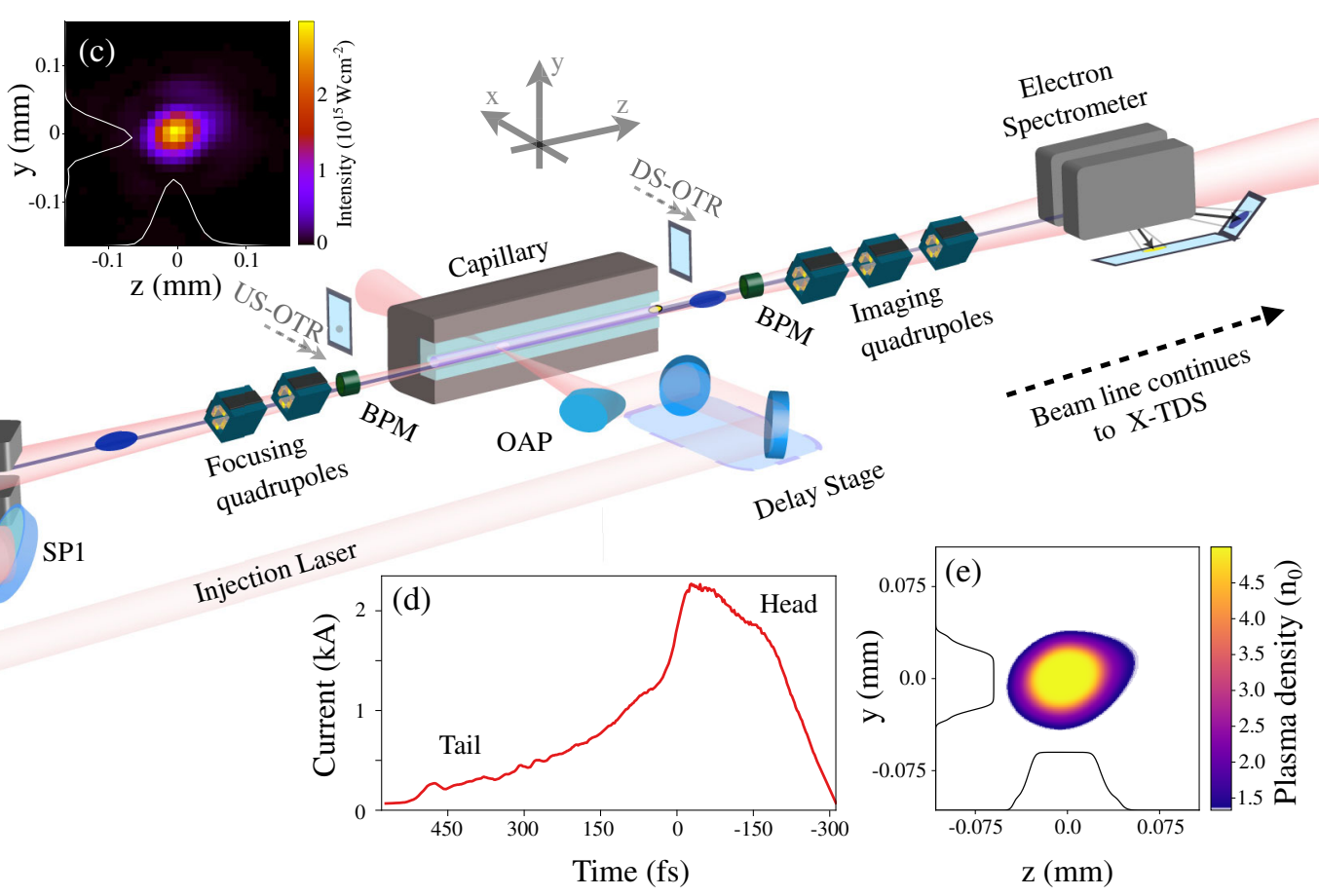

FIG. 1. Schematic layout depicting the key elements of the experimental setup at FLASHFORWARD. (a) Laser and electron beamline layout: P is the pick-off mirror for the transverse injection laser beam, SP1 and SP2 are spherical mirrors focusing the longitudinal ionization laser and OAP is the off axis parabola focusing the injection laser. The BPMs are shown; US-OTR and DS-OTR represent the upstream and downstream optical-transition-radiation screens, respectively. These can be moved into the beam path as alignment diagnostics. Foci for the (b) ionization and (c) injection lasers on the US-OTR are shown. (d) is the measured current profile of the drive beam. A side view of the plasma density calculated in the injection region from (c) is displayed in (e) (normalized to the background plasma density $n_{e}$ ). The plasma density is calculated from ADK ionization rates [50,51]. Black lines in (e) and white lines in (b,c) are axial projections.

encouraged by numerical studies showing witness beams with sub- $\mu \mathrm{m}$ transverse normalized emittances [31-34] and five-dimensional brightness values of up to $10^{19} \mathrm{Am}^{-2}$ $\operatorname{rad}^{-2}$ [34]. Furthermore, three-dimensional shaping of ramp gradients via laser ionization $[17,18]$ enables extended control over the injection process [35] and correspondingly the witness-beam phase space. At a constant plasma density $n_{e}$, the wakefield phase velocity is equal to the drive beam's velocity $v_{d}$, and close to the vacuum speed of light $c$. Therefore, witness beams remain phase stable over long distances such that energy gains are proportional to drivebeam energy, plasma length, and the transformer ratio [9,24]. The near-luminal phase velocity also precludes injection of electrons forming the boundary of the plasma wake. To realize injection, a negative plasma-density gradient along the propagation direction of the driver is required in order to locally reduce the phase velocity of the wake. The phase velocity in such a gradient is

$$
v_{\phi}=v_{d}\left(\frac{1}{2 n_{e}} \frac{\partial n_{e}(z)}{\partial z} \xi+1\right)^{-1}
$$

where $\xi=z-c t$ is the longitudinal coordinate in the comoving frame, $z$ is the longitudinal coordinate in the laboratory frame, and $t$ is time. Toward the back of the wake, the phase velocity decreases, allowing fast plasma electrons to rephase into the accelerating region, where they rapidly attain relativistic speed and form a witness beam that is subsequently accelerated in the wakefield. In laser-driven plasma accelerators [36-38], DDI has been applied with excellent control over witness-beam energy $[36,39]$ and with injection probabilities of up to $99 \%$ [40]. In combination with the advantages of PWFAs, it is, thus, an ideal candidate for a reliable beam-driven plasma cathode. Assessing the efficacy of such a device forms the main topic of this work.

This paper describes experiments carried out at FLASHFORWARD [41,42]—a beamline in the FLASH facility $[43,44]$ dedicated to plasma-wakefield research. It reports on the experimental realization of a stable and controlled internal injection process in PWFA, utilizing a laser-induced density downramp. As such, it represents the first demonstration of a beam-driven plasma cathode that can be reliably operated.

\section{EXPERIMENTAL SETUP}

Figure 1 depicts the experimental setup at FLASHFORWARD. Drive beams with a charge of $(790 \pm 4) \mathrm{pC}$, an energy of $(1116 \pm 6) \mathrm{MeV}$, and a peak current of $2.1 \mathrm{kA}$ [see Fig. 1(d)] were used to drive a plasma wake inside a capillary 
plasma source. The drive beam, with a transverse rms normalized emittance of $\epsilon_{x, y}=(14.0,5.3) \mu \mathrm{m}$, was focused at the capillary entrance by four quadrupoles. Using a combination of two cavity beam-position monitors (BPM) [45] and a spectrometer object-plane scan, the spot size was determined to be $\sigma_{x, y}^{\mathrm{d}}=(25.5 \pm 1.6) \mu \mathrm{m}$ and $(17.1 \pm$ 0.8) $\mu \mathrm{m}$ [46]. Well-resolved characterization of the longitudinal phase space on an X-band transverse-deflection structure [47-49] downstream of the interaction point provided a precise measurement of the drive-beam current profile shown in Fig. 1(e).

Plasma was generated via tunneling ionization inside the constant-gas-flow capillary [36,52,53]. Two separate laser pulses-referred to as the ionization pulse and injection pulse-propagated collinearly and perpendicularly, respectively, to the electron beam axis. The capillary had a total length of $50 \mathrm{~mm}$ with a circular access port for the transverse injection laser located $20 \mathrm{~mm}$ downstream of the entrance, leaving a total acceleration length of $30 \mathrm{~mm}$.

Uncertainty about the plasma length and width was identified in previous work as stability limiting [22]. By restricting the high-density plasma to the capillary length and choosing a large spot size for the ionization laser, these contributions to jitters were greatly reduced. With an $(x \times y)$ vacuum spot size of $(333 \times 461) \mu \mathrm{m}$ full width at half maximum (FWHM) [see Fig. 1(b)] and a meter-scale Rayleigh length, the ionization laser was able to ionize over at least the length of the capillary and form a plasma column sufficiently wide to contain the plasma wake. The plasma-density profile can be expected to follow the gasdensity profile with a taper developing toward the end of the capillary due to ionization defocusing (for details, see the Appendix). The injection laser was focused much more tightly than the ionization laser to a $(z \times y)$ spot size of $(57 \times 48) \mu \mathrm{m}$ FWHM [see Fig. 1(c)] and reached significantly higher intensity than the ionization laser to ionize more strongly bound electrons of the 2:1 helium-argon gas mixture. Thus, combined laser ionization can form a sharp density spike to initiate DDI, surrounded by a plateau region where the witness beam is accelerated. By comparing the measured drive-beam energy loss to three dimensional particle-in-cell (PIC) simulations, conducted with the OSIRIS code [54], the plateau plasma density was determined to be $1.4_{-0.3}^{+0.4} \times 10^{16} \mathrm{~cm}^{-3}$.

Spatial alignment between the laser arms and the electron beam was achieved with screens reflecting optical transition radiation at the injection point and downstream of the capillary. Both injection and ionization laser arms were derived from the same 25 TW Ti:sapphire laser system, synchronized to the FLASH electron beam at the 100femtosecond level [55] and compressed to a FWHM pulse duration of $(40 \pm 3)$ fs as measured in the injectionlaser beamline. The laser-ionized plasma on picosecond timescales follows the plasma shape determined by the intensity-distribution of the laser, until the hydrodynamic expansion dominates on the nanosecond timescale [56,57]. To avoid the regime of hydrodynamic expansion, the relative time-of-arrival was set such that the injection laser arrives $20 \mathrm{ps}$ and the injection laser $42 \mathrm{ps}$ earlier than the electron beam, as measured by delay-sensitive beam-heated plasma radiation [58].

The witness-beam charge was determined from excess charge measured as the difference between a cavity BPM [45] directly downstream and a toroidal current transformer (toroid) upstream of the interaction chamber with a combined resolution of $1 \mathrm{pC}$ [59]. As dark current originating from the FLASH linear accelerator can be defocused or absorped by the plasma cathode, the uncertainty in the excess-charge measurement can reach up to $5 \mathrm{pC}$. Simultaneously with the excess charge-measurement, detailed spectral and spatial information about the witness beams was retrieved from a LANEX scintillating phosphor screen as part of an imaging electron spectrometer capable of profiling electron beams from a few tens of $\mathrm{MeV}$ to $2.4 \mathrm{GeV}$.

\section{STABLE WITNESS-BEAM FORMATION}

The first investigation explored how the witness beams depended on the presence of the injection laser. This is shown in Fig. 2. Panel (a) depicts the energy spectrum of the witness beam over 500 consecutive shots at a spectrometer imaging energy of $45 \mathrm{MeV}$. The corresponding excess-charge values are plotted in panel (b). The injection laser was blocked and unblocked for 100 events at a time. Witness beams were only measured in the spectrometer if the injection laser was released. Outside the spectral range

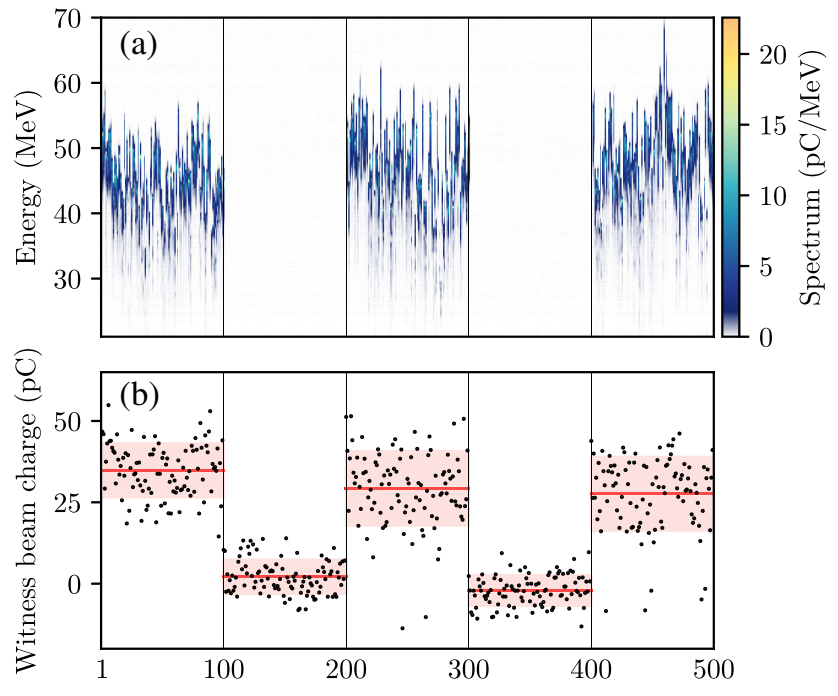

FIG. 2. (a) Energy spectra and (b) charge of the injected witness beam. The injection is switched off by blocking the injection laser for shot numbers 101-200 and 301-400. The mean value for each group of 100 shots is indicated by a solid line, while the rms variation of charge values is shown by a shaded area. 

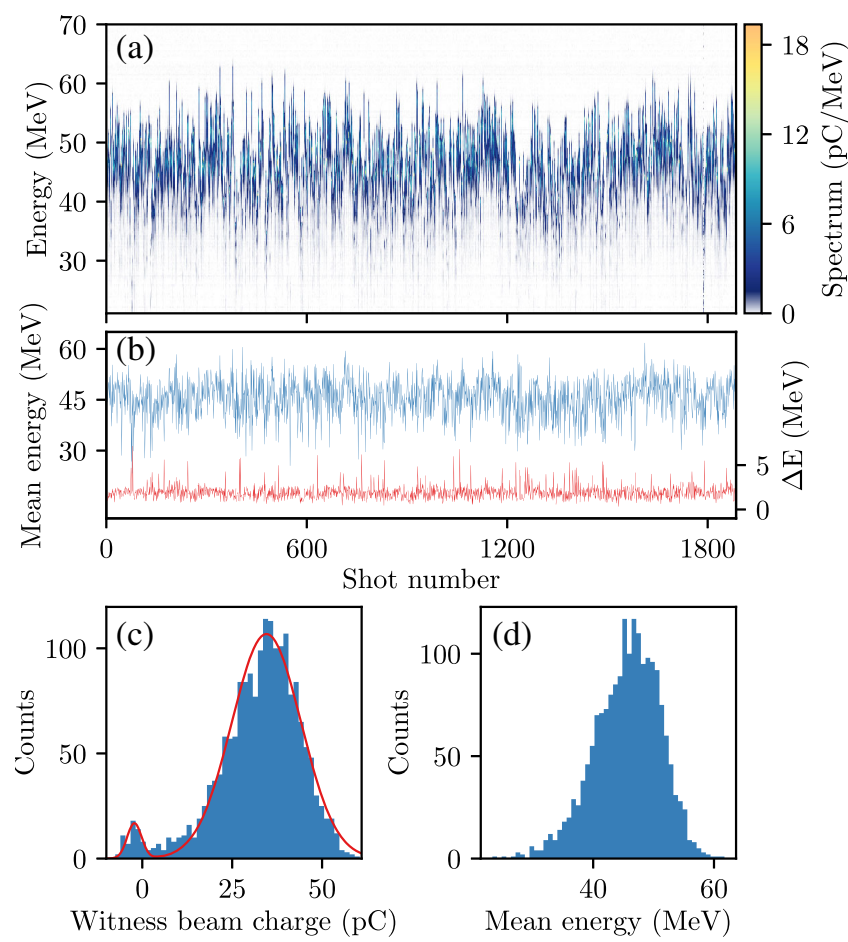

FIG. 3. (a) Waterfall plot of the witness-beam spectrum for 1885 consecutive shots. (b) The electron-beam mean energy (blue) and rms energy spread (red). (c) Histogram of the witnessbeam charge, measured from the calibrated spectrometer screen with a double-Gaussian fit shown in red. (d) Histogram of the witness beam mean energy. Each histogram has 50 bins.

of the plot, no witness-beam signal was observed. An excess charge of $Q_{\text {on }}=(32.1 \pm 9.6) \mathrm{pC}$ was detected when the injection laser was unblocked. The equivalent value when the injection laser was switched off was $Q_{\text {off }}=(0 \pm 5) \mathrm{pC}$. Both distributions are clearly separated by more than $2 \sigma$, unambiguously confirming that the witness-beam formation is triggered by the injection laser and DDI is established as the sole mechanism at play.

Next, a dataset of 1885 consecutive shots taken at a repetition rate of $2 \mathrm{~Hz}$ and recorded over a time span of approximately $15 \mathrm{~min}$ was used to explore the reliability of the plasma cathode. A waterfall plot of the projected witnessbeam energy spectra is shown in Fig. 3(a). No drift in spectral properties is observed. Figure 3(b) shows the evolution of the mean energy and rms energy spread. An injected witness beam was measured for $(95.4 \pm 2.5) \%$ of the shots, represented by the two distributions visible in Fig. 3(c). Analysis of these distributions yields a mean witness charge and energy of $(33 \pm 10) \mathrm{pC}$ and $(45 \pm 5) \mathrm{MeV}$, respectively, [see Fig. 3(c) and (d)] with an rms relative energy spread of $\left\langle\frac{\Delta E}{E}\right\rangle=4.4 \%$. Furthermore, no correlation between the witness beam charge or energy and the laser timing was detected up to $\approx 200$ ps relative time-of-arrival between drive beam and laser pulses.

\section{SENSITIVITY TO LASER ENERGY AND INJECTION-LASER ALIGNMENT}

The high injection probability and repeatability of the plasma cathode permit multishot characterization of the witness beam. For instance, variations of mean witnessbeam charge, energy, and energy spread as a function of on-target ionization-laser energy are plotted in Fig. 4(a-c). Analysis of the correlation coefficients yields, $\rho_{\text {Charge }}=$ $0.08, \rho_{\text {Energy }}=0.34$, and $\rho_{\Delta E / E}=0.12$, revealing a weak influence on witness-beam charge and thus indicating that the downramp-gradient did not vary strongly with laser energy. However, the influence on the witness-beam energy is much more pronounced. The relationship between laser energy and witness-beam energy is illustrated more clearly by systematically varying the laser energy over a broader range. The total laser energy was scanned in an on-targetenergy range of 44-96 $\mathrm{mJ}$ for the ionization laser and a corresponding range of $3.5-7.7 \mathrm{~mJ}$ for the injection laser. Figure 4(d) shows a nearly linear correlation between laser energy and the witness-beam energy. The slope extracted from the data translates the measured rms laser-energy jitter of $1.5 \%$ to a contribution of $3.9 \%$ rms to the energy jitter of the witness beam.

This contribution is sufficiently small that the effective accelerating field along the $30 \mathrm{~mm}$ acceleration length can be fine-tuned between 1.3 and $2.7 \mathrm{GV} / \mathrm{m}$ by varying the laser energy. The observed correlation can likely be explained by a higher plasma density throughout the

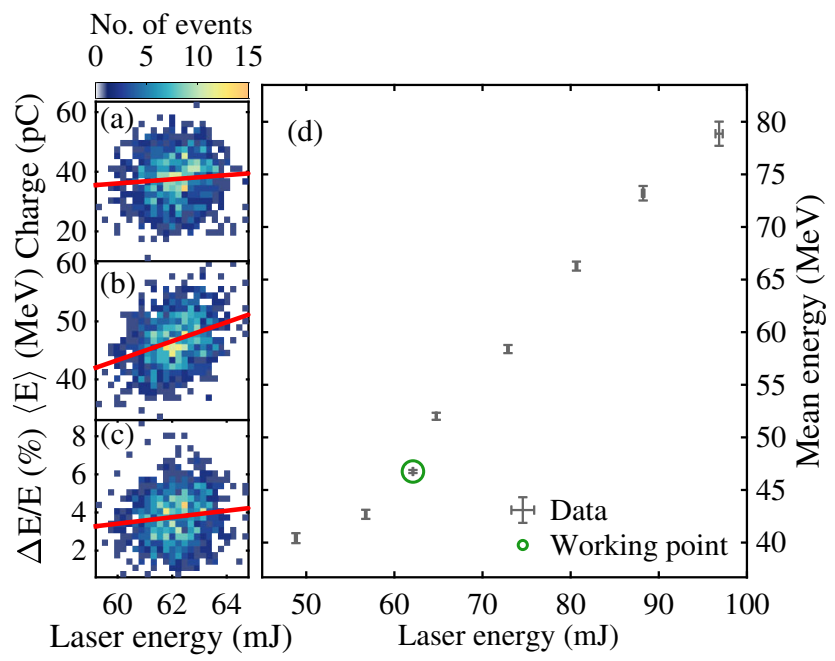

FIG. 4. (a) Correlation plots between on-target ionization-laser energy and charge (a), mean energy (b), and relative energy spread (c) for the injected witness beams in Fig. 3(a). Red lines indicate a linear fit through the data points. (d) Results of a systematic laser-energy scan highlighting the relationship between witness-beam energy and on-target laser energy. Error bars indicate the standard error. The working point for the data presented in this paper is indicated by a green circle. 
accelerating part of the plasma cathode from ionization of more strongly bound electrons at higher laser intensities, resulting in increased accelerating gradients. Plasma formation is decoupled from wake excitation because the driver does not ionize the plasma by itself. Therefore, any change in witness-beam properties is a result of a change to the plasma-density distribution. Energy-jitter in the witness beam can thus be further reduced e.g., by designing laser systems with lower energy jitter. If the argon in the gas mixture were to be replaced with a different gas e.g., hydrogen, the effect of ionization defocusing can be minimized and ionization along the gas-filled capillary source could more easily saturate, reducing the sensitivity to the laser energy at the cost of fine control over the witness-beam energy.

While witness-beam energy can be tuned by manipulating the laser energy, the injected charge shows a low sensitivity to laser energy; however, the relative position of the injection laser and electron beam has a much stronger influence. The relative $y$-position jitter between laser and electron beam is expected to be the predominant influence on the witness-beam charge, while other variations can be assumed to have a negligible effect. Variations in $z$ position of the laser of $\sigma_{z}^{\mathrm{inj}}=3.4 \mu \mathrm{m}$ only influence the injection position along the electron-beam axis, which only changes the acceleration length not the injection process. This gives a maximum contribution to the final energy jitter of $\approx 10 \mathrm{keV}$.

Similarily, the $x$-position jitter of the electron-beam waist $\sigma_{x}^{e}=1.0 \mu \mathrm{m}$ is much smaller than the Rayleigh length of the injection-laser focus and is therefore also considered to be negligible. Figure 5 shows the measured excess charge as a function of injection-laser position in $y$ relative to the electron-beam axis. Every data point plotted in Fig. 5 represent an average of 20 events, with the error on the measured charge given by the rms variation of the witnessbeam charge. Error bars on the position, $\sigma_{y}^{e, \text { inj }}=4.5 \mu \mathrm{m}$,

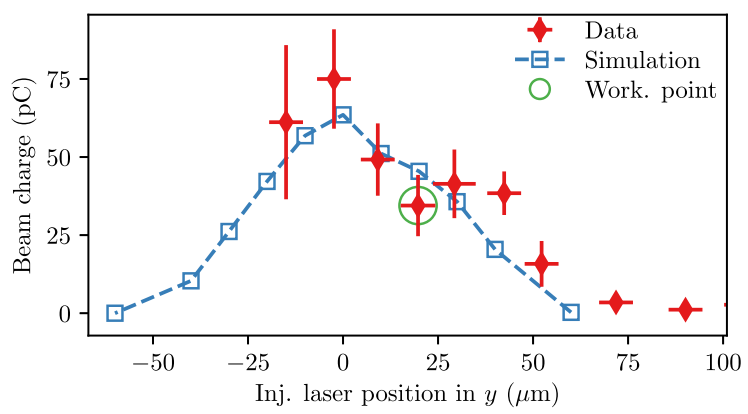

FIG. 5. Effect of centroid laser-spot positioning with respect to the electron beam. The laser position was measured on the upstream OTR camera while the charge was measured on the electron spectrometer screen (points with error bars). Results are compared to simulation results, shown as squares with the plasma profile modeled as shown in Fig. 1(e). The working point for the data presented in this paper is indicated by a green circle. were constant throughout the dataset and originated from two main sources: the rms $y$-position jitter of the electron beam at the injection position, $\sigma_{y}^{e}=1.4 \mu \mathrm{m}$, measured by two cavity BPMs [45] around the plasma chamber, and the rms variation of the laser-centroid $y$ position, $\sigma_{y}^{\mathrm{inj}}=4.2 \mu \mathrm{m}$, measured at the focus.

A comparison between the experimental data and a PIC-simulated offset scan is plotted in Fig. 5, based on the measured plasma profile [see Fig. 1(e)]. Here, $0 \mu \mathrm{m}$ corresponds to the highest simulated injected charge. The relative offset between the electron beam and injection laser could not be measured to sub-spot-size accuracy. Rather, it was determined by fitting the simulation-based model to the data. The data presented in Figs. 2-4 were taken at the working point indicated in Fig. 5 i.e., $24 \mu \mathrm{m}$ offset from the center of the injection plasma. Both simulations and experimental data show that the witness-beam charge can be maximized by optimizing positioning in $y$. Furthermore, the slope of the simulation-based model gives an estimate of the charge variation resulting from $y$-position jitter of $6.9 \mathrm{pC}$. Therefore, charge variation in the witness bunch can be explained predominantly by the position jitter between the electron beam and the injection laser, which is dominated by the laser pointing jitter. Such jitter would be eliminated e.g., by using an injection laser with a spot size that is wider than the plasma wake and has similar intensity. Focusing optics that produce asymmetric foci will be considered for this purpose in the future.

\section{WITNESS-BEAM EMITTANCE AND DIVERGENCE}

The brightness of a bunch of particles is dependent on its transverse emittance. Emittance-measurement techniques are typically averaged over many shots and, therefore, require stable and reproducible sources. In the past, this has not been possible with beams internally injected into PWFAs due to the previous instability of the plasma cathode. Instead, single-shot methods were utilized that e.g., rely on assumptions on the beta-function of the beam [22] or an energy-independent beam-slice emittance to take advantage of large energy spreads and the chromaticity in quadrupole focusing [19]. Such methods will, however, be increasingly difficult to apply with envisioned future high-quality beams with low-energy spread [60] and low divergence. In that case, multishot methods, as commonly used in accelerators, will be more adequate. The high stability described in this paper made a quadrupole scan possible, enabling the first multishot measurement of the injected bunch's transverse emittance.

Figure 6(a) shows the results of such a scan, providing the transverse beam size at the corresponding object plane $s$. In the absence of focusing forces, a witness beam with transverse geometric emittance $\varepsilon_{x}$ and a beta function $\beta^{*}$ at its virtual source point $s_{0}$ will expand outside the plasma such that it has a transverse rms beam size of 


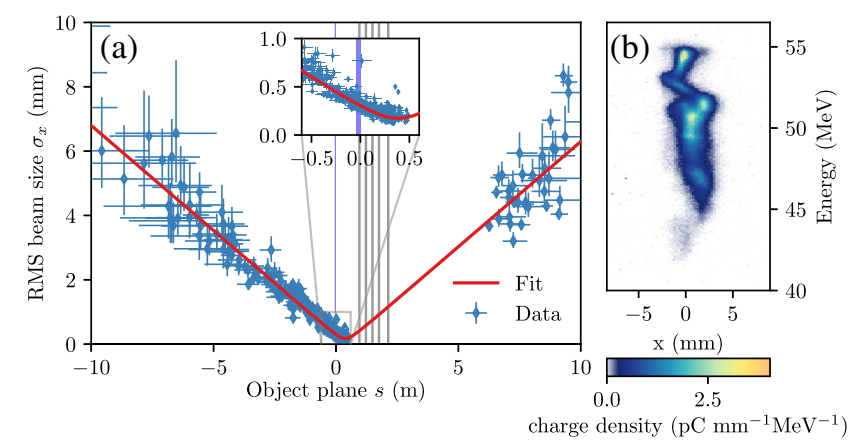

FIG. 6. (a) Scan of the imaging quadrupole focusing strength. Each data point represents a single event with an injected witness beam. Transverse beam sizes on the spectrometer screen and mean Lorentz factor $\langle\gamma\rangle$ were calculated from the spectrometer signal. From the quadrupole strength and $\langle\gamma\rangle$, the object plane $s$ and the magnification were calculated to extract $\sigma_{x}(s)$ at the corresponding object plane. Error bars on $\sigma_{x}$ and $s$ were calculated analogously based on the rms energy spread. The exit of the capillary (shown as a light blue line in (a) and the inset) is at $s=0$. Object planes in the range between $s=0.5 \mathrm{~m}-5.9 \mathrm{~m}$ lie too close to the quadrupoles (shown as gray lines) to be imaged. (b) An example of a witnessbeam signal as measured on the spectrometer screen.

$$
\sigma_{x}(s)=\sqrt{\varepsilon_{x}\left(\beta^{*}+\frac{\left(s-s_{0}\right)^{2}}{\beta^{*}}\right)}
$$

at longitudinal position $s$. Fitting Eq. (2) to the data gives a divergence of $\theta=(0.65 \pm 0.01) \mathrm{mrad}$ and $s_{0}=(381 \pm 6) \mathrm{mm}$.

The fact that $s_{0}$ is measured $381 \mathrm{~mm}$ downstream of the capillary exit is likely an effect of a weak plasma wake, driven in the laser-ionized low-density gas transition between the capillary exit and the vacuum. According to fluid simulations, the on-axis gas density is expected to drop at the capillary exit by two orders of magnitude over a distance of $\approx 40 \mathrm{~mm}$, at which point it approaches the measured ambient gas density in the experimental chamber of $10^{14} \mathrm{~cm}^{-3}$. This gas would be ionized because of the long Rayleigh length of the ionization laser. Plasma wakes driven in such a low-density plasma continue to focus witness beams for several centimeters beyond the capillary and continuously increase their beta functions [61], possibly explaining the particularly low divergence observed in this experiment.

The transverse normalized emittance,

$$
\epsilon_{n}(s)=\langle\gamma\rangle \sqrt{\left\langle\frac{\Delta E}{E}\right\rangle^{2} \sigma_{x}^{2}{\sigma_{x^{\prime}}}^{2}+\epsilon_{x}^{2}},
$$

depends on the $s$-dependent rms beam divergence $\sigma_{x^{\prime}}$, spot size $\sigma_{x}$, rms energy spread, and mean Lorentz factor $\langle\gamma\rangle$. Therefore, beams from plasma cathodes with high-energy spread and high divergence can suffer from emittance growth after exiting the plasma [62]. The measurement described here gives an emittance at the plasma exit of $\varepsilon_{x}^{n}\left(s_{0}\right)=(9.3 \pm 0.3) \mu \mathrm{m}$, increasing by less than $0.2 \%$ before reaching the first quadrupole at a distance of $0.658 \mathrm{~m}$. An example of the witness-beam signal measured on the spectrometer screen is shown in Fig. 6(b). The visible oscillatory structure can be interpreted as transverse centroid oscillations of longitudinal witness-beam slices that have probably contributed to an increase in projected emittance during acceleration.

\section{CONCLUSION}

This paper demonstrates a plasma cathode with stability at a level unprecedented in the field of beam-driven plasma injectors. The high degree of stability enabled detailed studies of the plasma cathode, revealed dependencies between witness-beam and plasma parameters, and enabled the first multishot emittance and divergence measurement of an internally injected witness beam. The presented results are compatible with very low emittance growth at the extraction from the plasma. Effective accelerating gradients, which can be precisely tuned in the range 1.3-2.7 GV/m, were demonstrated. Sources of charge and energy jitter were identified, and mitigation strategies were proposed for future applications. The setup described here will enable future optimization of the working point to reach lower initial emittance e.g., by improving the overlap between injection-laser and electron beam, symmetric focusing of the drive-beam or via manipulation of the drive-beam current profile to reduce offset-oscillations in the witness beam. Furthermore, the setup will enable studying beam propagation after the exit of plasma stages, which is of high importance for the transport of lowemittance beams from plasma cathodes in general. The presented results constitute a significant step toward stable, controllable beam-driven plasma cathodes and brightnessconverter stages, which are of great interest for nextgeneration photon-science and particle-physics facilities.

\section{ACKNOWLEDGMENTS}

The authors thank M. Dinter, S. Karstensen, K. Ludwig, F. Marutzky, A. Rahali, V. Rybnikov, A. Schleiermacher, and S. Kottler, as well as the FLASH team, MVS group, divisions of high energy $(\mathrm{FH})$, and machine $(\mathrm{M})$ physics at DESY for their support. We also thank the OSIRIS consortium (IST/ UCLA) for access and support with the OSIRIS threedimensional code; M. Kirchen and S. Jalas, from the University of Hamburg, for making the pipeline-analysis software available. We gratefully acknowledge the use of High-Performance Cluster (Maxwell) at DESY and Gauss Centre for Supercomputing (GCS) e.V. resources. GCS has supported this project by providing computing time through the John von Neumann Institute for Computing (NIC) on the GCS Supercomputer JUWELS at Jülich Supercomputing Centre (JSC). B. H. and L. B. acknowledge the support of the European Research Council (ERC) under the European 
Unions Horizon 2020 research and innovation programme (NeXource, ERC Grant agreement No. 865877).

\section{APPENDIX A: LASER IONIZATION}

Ionization laser and injection laser were separated with a $0.5^{\prime \prime}$ pick-off mirror that was inserted into the laser path to reflect the central part of the $34 \mathrm{~mm}$-diameter laser beam into the injection-laser beamline. As depicted in Fig. 1(a), the remaining part of the laser continued to propagate along the ionization-laser arm. Focusing of the ionization laser was achieved by two spherical mirrors with a resulting effective focal length of $18 \mathrm{~m}$. An $f / 51$ off axis parabola focused the injection laser perpendicular to the electron-beam axis through a fused silica window. To minimize nonlinear dispersion that can deteriorate the quality of the focal spot or pulse length, the window thickness was chosen to be $1.5 \mathrm{~mm}$ only, resulting in a maximal B-integral of 0.27 . The intensity of the injection laser was set to be significantly higher than that of the ionization laser such that the latter ionized argon up to its second level whilst the former was capable of additionally ionizing the third ionization level of argon and the first of helium (see Table I). At the same time, the required intensities are sufficiently low to avoid significant ponderomotive heating of the plasma electrons [63].

\section{APPENDIX B: ALIGNMENT AND SYNCHRONIZATION}

The precise relative time-of-arrival between electronbeam, ionization laser, and injection laser was adjusted utilizing plasma-afterglow observation of beam-heated plasmas. The recombination light from the plasma after the passage of an electron beam with currents of order kA can be used to synchronize lasers to electron beams to within a few femtoseconds [58]. This effect was used to synchronize the two laser arms to the drive beam. For this measurement, the experimental chamber was filled with argon gas to 0.05 mbar and a plasma was ionized by the corresponding lasers with the capillary removed. Figure 7 shows the time-of-arrival (TOA) dependence of plasma light collected by a charge-coupled device camera viewing from the top for each of the laser arms-examples of

TABLE I. Relevant ionization energies [64] and the corresponding laser intensities for a tunnel ionization rate [51] of $1 \mathrm{fs}^{-1}$ at a laser wavelength of $800 \mathrm{~nm}$.

\begin{tabular}{lcc}
\hline \hline $\begin{array}{l}\text { Ionization } \\
\text { level }\end{array}$ & $\begin{array}{c}\text { Ionization energy } \\
(\mathrm{eV})\end{array}$ & $\begin{array}{c}\mathrm{I}\left(\mathrm{W}_{\mathrm{ADK}}=1 \mathrm{fs}^{-1}\right) \\
\left(\mathrm{W} / \mathrm{cm}^{2}\right)\end{array}$ \\
\hline Ar-I & 15.76 & $4.6 \times 10^{14}$ \\
Ar-II & 27.63 & $1.0 \times 10^{15}$ \\
Ar-III & 40.74 & $2.1 \times 10^{15}$ \\
He-I & 24.59 & $2.2 \times 10^{15}$ \\
He-II & 54.42 & $1.2 \times 10^{16}$ \\
\hline \hline
\end{tabular}

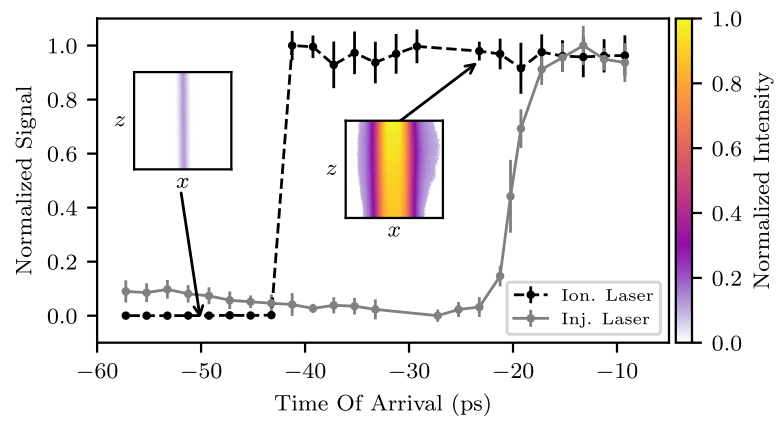

FIG. 7. Normalized integrated plasma-afterglow signal as a function of the time-of-arrival (TOA) between the drive beam and the ionization (Ion.) and injection (Inj.) lasers; $t=0$ is defined as the TOA of the electron beam. Inset are examples of the signal enhancement due to electron-beam-induced heating of the plasma formed by the ionization laser.

camera images are depicted as insets. The visible transitions in Fig. 7 determine the TOA delay settings with respect to the electron beam. Once the lasers were set up, the capillary was positioned to maximize laser transmission through the capillary holes.

\section{APPENDIX C: PIC SIMULATIONS}

The gas flow inside and outside the capillary was modeled with COMSOL and ANSYS. To simulate the plasma generation by the ionization laser, the resulting model of the gas-density profile and measurements of the laser parameters served as input into a numerical study conducted with the code FBPIC [65]. These studies showed that the plasma density can develop a taper toward the downstream end of the capillary due to ionization defocusing of the ionization laser. Similar simulations confirmed that in the case of the injection laser, the effect of the interaction with plasma onto the laser-pulse is sufficiently small and the shape of the plasma-density spike can be calculated from the intensity-distribution as measured in vacuum. The injection and acceleration of the witness beam was finally modeled with the three-dimensional PIC simulation code OSIRIS [54]. Simulations were performed with a comoving window of $(460 \times 400 \times 400) \mu \mathrm{m}$ in $(z, x, y)$ with $(256 \times 256 \times 256)$ cells. A resolution convergence test was conducted. The drive-beam current profile, charge, and spot size were modeled based on measurements. The number of macroparticles per cell (PPC) for the drive beam was 8 . While the PPC for the background plasma electrons was 1 , the PPC was increased to 8 in a radius of $100 \mu \mathrm{m}$ around the density downramp to resolve the physics of the injection process more accurately. The modeled plasma-density distribution was assumed with a plateau density of $1.4 \times 10^{16} \mathrm{~cm}^{-3}$. Since the detailed structure of the taper can vary with laser parameters and gas density, the knowledge of the precise on axis plasma density decreases with laser-propagation distance. As a result, the OSIRIS 


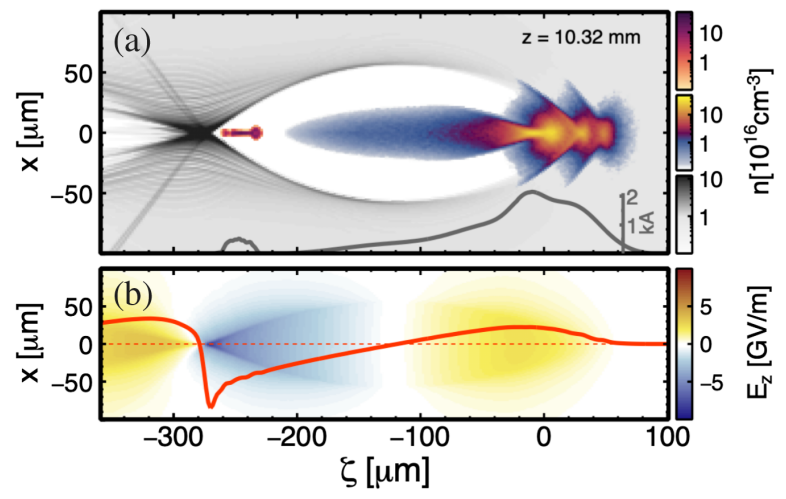

FIG. 8. Simulation snapshot during the witness-beam acceleration: Cut through the plasma wake with electron densities (a) of witness beam (top right-hand colorbar), drive-beam (central colorbar), background plasma (bottom colorbar), and longitudinal current profile (solid gray line). (b) Longitudinal electric field as a central cut and an on axis one-dimensional projection (solid red line).

simulations are assumed to only model the experiment with sufficient accuracy until shortly after the injection and thus results are shown at a longitudinal position of $z=10.32 \mathrm{~mm}$ downstream of the injection-laser focus. A snapshot of the simulated acceleration process can be seen in Fig. 8 and the slice properties and longitudinal phase space of the witness beam are shown in Fig. 9. The injection laser was positioned $(23.5 \pm 4.5) \mu \mathrm{m}$ off axis with respect to the electron beam and varied to simulate the offset scan.

\section{APPENDIX D: EMITTANCE MEASUREMENT}

To calculate the datapoints shown in Fig. 6, the object plane and magnification was evaluated depending on energy and focusing strength of the imaging quadrupoles. This calculation was performed for the mean energy, upper standard-deviation limit and lower standard-deviation limit,

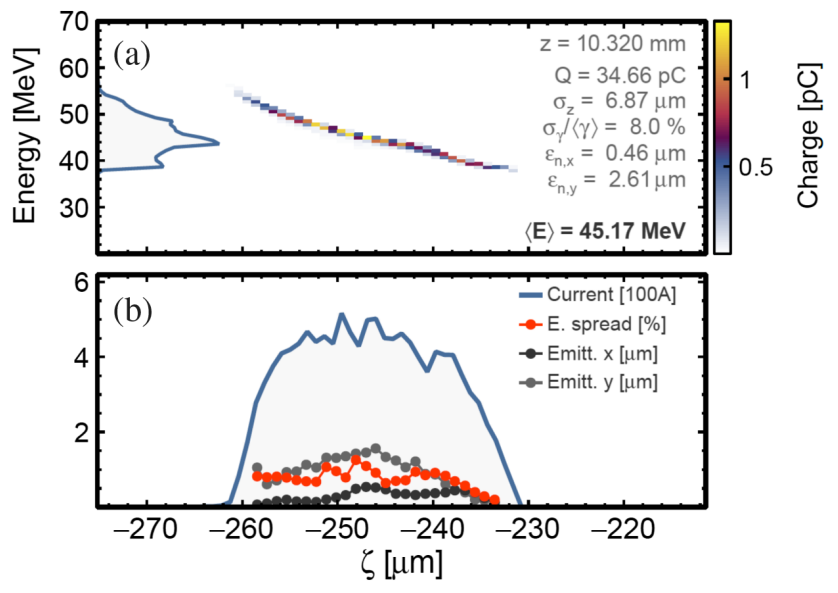

FIG. 9. Longitudinal phase space of the simulated witnessbeam parameter (a) and slice parameters (b) after $10.32 \mathrm{~mm}$ of acceleration.

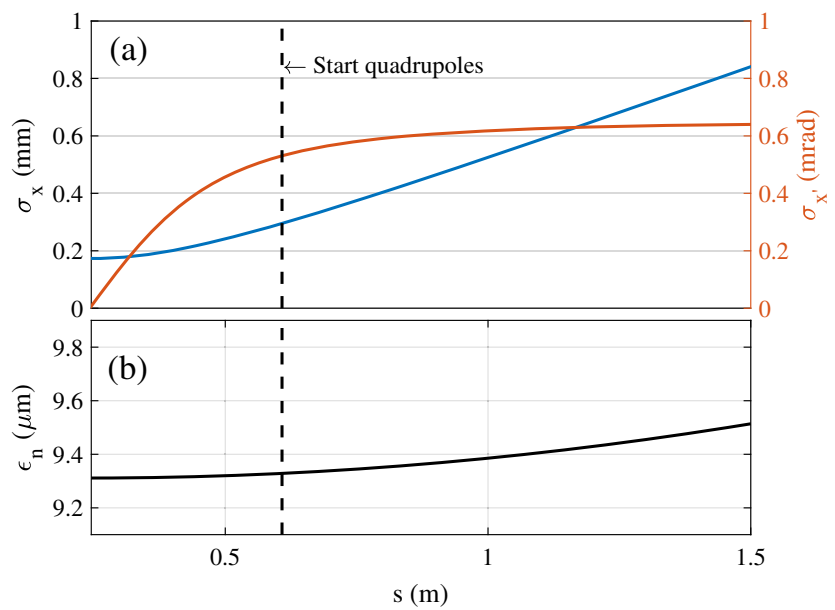

FIG. 10. (a) Fit result of measured waist of witness-beam and corresponding $s$-dependent divergence. (b) Emittance-growth in the drift-space downstream of the plasma cathode.

giving, respectively, the mean value and error limits for $s$ and $\sigma_{x}$ for each data point. The measurement samples the virtual waist of the free-space propagation of the witness beam outside the plasma, which follows Eq. (2). An equation $\sigma_{x}(s)=\sqrt{A+B\left(s-s_{0}\right)^{2}}$, with fit parameters $A, B$, and $s_{0}$ was fitted to the data. From $A=\beta^{*} \epsilon_{x}$ and $B=\frac{\epsilon_{x}}{\beta^{*}}$, the geometric emittance $\epsilon_{x}$ and the waist beta function $\beta^{*}$ were calculated.

It can be shown that the normalized emittance of electron beams with nonzero energy spread can increase in the driftspace downstream of the waist [62]. For relativistic beams $\left(\frac{v}{c} \approx 1\right)$ under the assumption that $\gamma$ is independent of $x$ and $x^{\prime}$, this effect is described by Eq. (3). Figure 10 illustrates the postplasma emittance growth of the measured plasma cathode. The spot size $\sigma_{x}(s)$ according to the fit results and $\sigma_{x^{\prime}}(s)=\frac{d \sigma_{x}(s)}{d s}$ is plotted in panel (a), where the divergence $\theta$ is the asymptotic limit of $\sigma_{x^{\prime}}(s)$ for large distances $s$. The corresponding growth in normalized emittance is plotted in Fig. 10(b) and shows that the measurements are compatible with a small increase of less than $1 \%$ over $1 \mathrm{~m}$ of propagation outside the plasma.

[1] J. B. Rosenzweig, D. B. Cline, B. Cole, H. Figueroa, W. Gai, R. Konecny, J. Norem, P. Schoessow, and J. Simpson, Phys. Rev. Lett. 61, 98 (1988).

[2] P. Chen, J. M. Dawson, R. W. Huff, and T. Katsouleas, Phys. Rev. Lett. 54, 693 (1985).

[3] J. B. J. B. Rosenzweig, B. Breizman, T. Katsouleas, and J. J. Su, Phys. Rev. A 44, R6189 (1991).

[4] T. Tajima and J. M. Dawson, Phys. Rev. Lett. 43, 267 (1979).

[5] R. D. Ruth, A. W. Chao, L. Morton, and P. B. Wilson, Part. Accel. 17, 171 (1985), https://cds.cern.ch/record/157249. 
[6] B. Cros, P. Muggli, C. Schroeder, S. Hooker, P. Piot, J. England, S. Gessner, J. Vieira, E. Gschwendtner, J.-L. Vay et al., arXiv:1901.10370.

[7] I. Blumenfeld, C. E. Clayton, F. J. Decker, M. J. Hogan, C. Huang, R. Ischebeck, R. Iverson, C. Joshi, T. Katsouleas, N. Kirby et al., Nature (London) 445, 741 (2007).

[8] M. Litos, E. Adli, W. An, C. I. Clarke, C. E. Clayton, S. Corde, J. P. Delahaye, R. J. England, A. S. Fisher, J. Frederico et al., Nature (London) 515, 92 (2014).

[9] G. Loisch, G. Asova, P. Boonpornprasert, R. Brinkmann, Y. Chen, J. Engel, J. Good, M. Gross, F. Grüner, H. Huck et al., Phys. Rev. Lett. 121, 064801 (2018).

[10] S. Corde, E. Adli, J. M. Allen, W. An, C. I. Clarke, C. E. Clayton, J. P. Delahaye, J. Frederico, S. Gessner, S. Z. Green et al., Nature (London) 524, 442 (2015).

[11] C. A. Lindstrøm, J. M. Garland, S. Schröder, L. Boulton, G. Boyle, J. Chappell et al., Phys. Rev. Lett. 126, 014801 (2021).

[12] R. Pompili, D. Alesini, M. Anania, M. Behtouei, M. Bellaveglia, A. Biagioni, F. Bisesto, M. Cesarini, E. Chiadroni, A. Cianchi et al., Nat. Phys. 17, 499 (2021).

[13] B. Hidding, G. Pretzler, J. B. Rosenzweig, T. Königstein, D. Schiller, and D. L. Bruhwiler, Phys. Rev. Lett. 108, 035001 (2012).

[14] F. Li, J. F. Hua, X. L. Xu, C. J. Zhang, L. X. Yan, Y. C. Du, W. H. Huang, H. B. Chen, C. X. Tang, W. Lu et al., Phys. Rev. Lett. 111, 015003 (2013).

[15] A. Martinez de la Ossa, J. Grebenyuk, T. Mehrling, L. Schaper, and J. Osterhoff, Phys. Rev. Lett. 111, 245003 (2013).

[16] Y. Wan, C. J. Zhang, F. Li, Y. P. Wu, J. F. Hua, C. H. Pai, W. Lu, Y. Q. Gu, X. L. Xu, C. Joshi, and W. B. Mori, Plasma Phys. Controlled Fusion 58, 034015 (2016).

[17] G. Wittig, O. Karger, A. Knetsch, Y. Xi, A. Deng, J. B. Rosenzweig, D. L. Bruhwiler, J. Smith, G. G. Manahan, Z. M. Sheng et al., Phys. Rev. ST Accel. Beams 18, 081304 (2015).

[18] G. Wittig, O. S. Karger, A. Knetsch, Y. Xi, A. Deng, J. B. Rosenzweig, D. L. Bruhwiler, J. Smith, Z. M. Sheng et al., Nucl. Instrum. Methods Phys. Res., Sect. A 829, 83 (2016).

[19] N. Vafaei-Najafabadi, W. An, C. Clayton, C. Joshi, K. Marsh, W. Mori, E. Welch, W. Lu, E. Adli, J. Allen et al., Plasma Phys. Controlled Fusion 58, 034009 (2016).

[20] G. Manahan, A. Deng, O. Karger, Y. Xi, A. Knetsch, M. Litos, G. Wittig, T. Heinemann et al., Phys. Rev. Accel. Beams 19, 011303 (2016).

[21] N. Vafaei-Najafabadi, K. A. Marsh, C. E. Clayton, W. An, W. B. Mori, C. Joshi, W. Lu, E. Adli, S. Corde, M. Litos et al., Phys. Rev. Lett. 112, 025001 (2014).

[22] A. Deng, O. S. Karger, T. Heinemann, A. Knetsch, P. Scherkl, G. G. Manahan, A. Beaton, D. Ullmann, G. Wittig, A. F. Habib et al., Nat. Phys. 15, 1156 (2019).

[23] E. Oz, S. Deng, T. Katsouleas, P. Muggli, C. D. Barnes, I. Blumenfeld, F. J. Decker, P. Emma, M. J. Hogan, R. Ischebeck et al., Phys. Rev. Lett. 98, 084801 (2007).

[24] T. Katsouleas, Phys. Rev. A 33, 2056 (1986).

[25] H. Suk, N. Barov, J. B. Rosenzweig, and E. Esarey, Phys. Rev. Lett. 86, 1011 (2001).

[26] R. J. England, J. B. Rosenzweig, and N. Barov, Phys. Rev. E 66, 016501 (2002).
[27] M. C. Thompson, J. B. Rosenzweig, and H. Suk, Phys. Rev. ST Accel. Beams 7, 011301 (2004).

[28] S. Bulanov, N. Naumova, F. Pegoraro, and J. Sakai, Phys. Rev. E 58, R5257 (1998).

[29] P. Tomassini, M. Galimberti, A. Giulietti, D. Giulietti, L. A. Gizzi, L. Labate, and F. Pegoraro, Phys. Rev. ST Accel. Beams 6, 121301 (2003).

[30] F. Albert, M.-E. Couprie, A. D. Debus, M. Downer, J. Faure, A. Flacco, L. A. Gizzi et al., New J. Phys. 23, 031101 (2021).

[31] J. Grebenyuk, A. M. de la Ossa, T. Mehrling, and J. Osterhoff, Nucl. Instrum. Methods Phys. Res., Sect. A 740, 246 (2014).

[32] A. Martinez de la Ossa, Z. Hu, M. J. V. Streeter, T. J. Mehrling, O. Kononenko, B. Sheeran, and J. Osterhoff, Phys. Rev. Accel. Beams 20, 091301 (2017).

[33] X. L. Xu, F. Li, W. An, T. N. Dalichaouch, P. Yu, W. Lu, C. Joshi, and W. B. Mori, Phys. Rev. Accel. Beams 20, 111303 (2017).

[34] C. Zhang, C.-K. Huang, K. A. Marsh, X. L. Xu, F. Li, M. Hogan, V. Yakimenko, S. Corde, W. B. Mori, and C. Joshi, Phys. Rev. Accel. Beams 22, 111301 (2019).

[35] D. Ullmann, P. Scherkl, A. Knetsch, T. Heinemann, A. Sutherland, A. Habib, O. Karger, A. Beaton, G. Manahan, A. Deng et al., arXiv:2007.12634.

[36] A. J. Gonsalves, K. Nakamura, C. Lin, D. Panasenko, S. Shiraishi, T. Sokollik, C. Benedetti, C. B. Schroeder, C. G. R. Geddes, J. Van Tilborg et al., Nat. Phys. 7, 862 (2011).

[37] J. Faure, C. Rechatin, O. Lundh, L. Ammoura, and V. Malka, Phys. Plasmas 17, 083107 (2010).

[38] K. Schmid, A. Buck, C. M. S. Sears, J. M. Mikhailova, R. Tautz, D. Herrmann, M. Geissler, F. Krausz, and L. Veisz, Phys. Rev. ST Accel. Beams 13, 091301 (2010).

[39] K. Swanson, H.-E. Tsai, S. Barber, R. Lehe, H.-S. Mao, S. Steinke, J. Van Tilborg, K. Nakamura, C. Geddes, C. Schroeder et al., Phys. Rev. Accel. Beams 20, 051301 (2017).

[40] A. Buck, J. Wenz, J. Xu, K. Khrennikov, K. Schmid, M. Heigoldt, J. M. Mikhailova, M. Geissler, B. Shen, F. Krausz et al., Phys. Rev. Lett. 110, 185006 (2013).

[41] A. Aschikhin, C. Behrens, S. Bohlen, J. Dale, N. Delbos, L. di Lucchio, E. Elsen, J.-H. Erbe, M. Felber, B. Foster et al., Nucl. Instrum. Methods Phys. Res., Sect. A 806, 175 (2016).

[42] R. D’Arcy, A. Aschikhin, S. Bohlen, G. Boyle, T. Brümmer, J. Chappell, S. Diederichs, B. Foster, M. J. Garland, L. Goldberg et al., Phil. Trans. R. Soc. A 377, 20180392 (2019).

[43] W. Ackermann, G. Asova, V. Ayvazyan, A. Azima, N. Baboi, J. Bähr, V. Balandin, B. Beutner, A. Brandt, A. Bolzmann et al., Nat. Photonics 1, 336 (2007).

[44] S. Schreiber and B. Faatz, High Power Laser Sci. Eng. 3, e20 (2015).

[45] D. Lipka, D. Nölle, M. Siemens, and S. Vilcins, in Proceedings of LINAC (JACoW, Tsukuba, 2010).

[46] C. A. Lindstrøm, R. D’ Arcy, M. J. Garland, P. Gonzalez, B. Schmidt, S. Schröder, S. Wesch, and J. Osterhoff, Phys. Rev. Accel. Beams 23, 052802 (2020).

[47] B. Marchetti, R. Assmann, B. Beutner, J. Branlard, F. Christie, R. D’Arcy, W. Decking, U. Dorda, J. Herrmann 
et al., in Proceedings of IPAC17 (JACoW, Copenhagen, 2017), pp. 184-187.

[48] R. D’Arcy, A. Aschikhin, P. Gonzalez Caminal, V. Libov, and J. Osterhoff, in Proceedings of the 9th International Particle Accelerator Conference (Joint Accelerator Conferences Website (JaCOW), Geneva, 2018).

[49] R. DArcy, V. Libov, and J. Osterhoff, in Proceedings of IBIC'16 (JACOW, Barcelona, 2016), pp. 760-763.

[50] D. L. Bruhwiler, D. A. Dimitrov, J. R. Cary, E. Esarey, W. Leemans, and R. E. Giacone, Phys. Plasmas 10, 2022 (2003).

[51] M. V. Ammosov, N. B. Delone, and V.P. Krainov, in Proceedings of SPIE 0664, High Intensity Laser Processes (1986), Vol. 0664, https://doi.org/10.1117/12.938695.

[52] A. J. Gonsalves, K. Nakamura, J. Daniels, C. Benedetti, C. Pieronek, T. C. De Raadt, S. Steinke, J. H. Bin, S. S. Bulanov, J. Van Tilborg, C. G. Geddes, C. B. Schroeder, C. Tóth, E. Esarey, K. Swanson, L. Fan-Chiang, G. Bagdasarov et al., Phys. Rev. Lett. 122, 084801 (2019).

[53] A. Butler, D. J. Spence, and S. M. Hooker, Phys. Rev. Lett. 89, 185003 (2002).

[54] R. A. Fonseca, L. O. Silva, F. S. Tsung, V. K. Decyk, W. Lu, C. Ren, W. B. Mori, S. Deng, S. Lee, T. Katsouleas, and J. C. Adam, in International Conference on Computational Science (Springer, Berlin, 2002), pp. 342-351.

[55] S. Schulz, I. Grguraš, C. Behrens, H. Bromberger, J. T. Costello, M. K. Czwalinna, M. Felber, M. C. Hoffmann, M. Ilchen, H. Y. Liu et al., Nat. Commun. 6 (2015).

[56] C. G. Durfee and H. M. Milchberg, Phys. Rev. Lett. 71, 2409 (1993).
[57] R. J. Shalloo, C. Arran, L. Corner, J. Holloway, J. Jonnerby, R. Walczak, H. M. Milchberg, and S. M. Hooker, Phys. Rev. E 97, 053203 (2018).

[58] P. Scherkl, A. Knetsch, T. Heinemann, A. Sutherland, A. F. Habib, O. Karger, D. Ullmann, A. Beaton, G. Kirwan, G. Manahan et al., arXiv:1908.09263.

[59] D. Lipka, A. Affeldt, R. Awwad, N. Baboi, R. Barret, B. Beutner, F. Brinker, W. Decking, A. Delfs, M. Drewitsch et al., in Proceedings of IBIC'16 (JACoW, Barcelona, 2016), pp. 14-19.

[60] G. G. Manahan, A. F. Habib, P. Scherkl, P. Delinikolas, A. Beaton, A. Knetsch, O. Karger, G. Wittig, T. Heinemann, Z.-M. Sheng et al., Nat. Commun. 8 (2017).

[61] K. Floettmann, Phys. Rev. ST Accel. Beams 17, 054402 (2014).

[62] M. Migliorati, A. Bacci, C. Benedetti, E. Chiadroni, M. Ferrario, A. Mostacci, L. Palumbo, A. R. Rossi, L. Serafini, and P. Antici, Phys. Rev. ST Accel. Beams 16, 011302 (2013).

[63] C. B. Schroeder, J. L. Vay, E. Esarey, S. S. Bulanov, C. Benedetti, L.-L. Yu, M. Chen, C. G. R. Geddes, and W. P. Leemans, Phys. Rev. ST Accel. Beams 17, 101301 (2014).

[64] A. Kramida, Y. Ralchenko, and NIST ASD Team, NIST Atomic Spectra Database Ionization Energies, 2020, https://physics.nist.gov/asd.

[65] R. Lehe, M. Kirchen, I. A. Andriyash, B. B. Godfrey, and J. L. Vay, Comput. Phys. Commun. 203, 66 (2016). 